\title{
What is the perfect nose? Lesson learnt from the literature*
}

\section{Anni Ding', Yuanpei Zhang²}

Academic Foundation Doctor, Imperial College Healthcare NHS Trust, London, United Kingdom

${ }^{2}$ Academic Foundation Doctor, Torbay and South Devon NHS Foundation Trust, London, United Kingdom
Rhinology Online, Vol 3: 25 - 30, 2020

http://doi.org/10.4193/RHINOL/20.012

*Received for publication:

February 24, 2020

Accepted: April 6, 2020

Published: May 2, 2020

\begin{abstract}
Background: The nose, as the central feature of the face, has a pivotal role in determining the overall proportion of the face, and is a topic of great interest in facial aesthetics.
\end{abstract}

Methodology: A literature review to elucidate a 'perfect' nose from an aesthetic stance.

Results: The width of the nose should be equal to the middle fifth based on neoclassical canon. The ideal mouth to nose width ratio conforms to the golden ratio. The ideal nose length $(\mathrm{RT})$ is $0.67 \mathrm{x}$ midfacial height. A straight dorsum with no supratip break or a straight dorsum reduced to a level of $2 \mathrm{~mm}$ below the tip creating a retroussé are both desirable. Numerous rules have been proposed with regards to ideal tip projection, Goode's rule is perhaps the most widely used - the ratio of the projection of the nose measured from the alar crease should be $0.55-0.60$ of the nasal dorsal length. The columella show should be $2-4 \mathrm{~mm}$. More recently, it has been suggested that a mathematically averaged nose is attractive.

Conclusion: Whilst many rules/ratios have been described, there's no universal standard for the 'perfect' nose. The neoclassical norms may no longer represent the ideal, and facial aesthetics may be inciting changes in the standard of beauty.

Key words: nose, nose deformities, nasal surgical procedures, nasal bone, nasal septum

\section{Introduction}

Mankind has always been fascinated with beauty and elucidating key elements that makes someone regarded as beautiful. The nose, as the central feature of the face, has a pivotal role in determining the overall balance and proportion of the face. It should be in harmony with the other facial features and allow them to be appreciated. Dating back as far as ancient Greek times, many rules and ratios have been proposed in an attempt to mathematically quantify the 'perfect' nose. Facial aesthetic practice, including rhinoplasty has seen an exponential growth in recent years, as a result, these geometrical rules have been drawn upon to help achieve the best cosmetic result ${ }^{(1)}$. This narrative review aims to examine the various canons described in the literature in trying to elucidate a 'perfect' nose from an aesthetic point of view.

\section{Function and anatomy of the nose}

The nose has a pyramidal shape, with the nasal root located cranially and continuous with the forehead. The apex of the nose ends inferiorly as the nasal tip. The dorsum of the nose connects the root and the tip. The nasal septum forms the central strut of the nose and separates the two nasal airways. The upper third of the nose is made up of a pair or nasal bones, the middle third comprises of stiffer paired upper lateral cartilages (ULC), through their attachment to the nasal bones, they provide structural support for the septum. The lower third comprises of a pair of softer lower lateral cartilages (LLC). The LLC are delicate and rests on the ULC for support. There is an overlap between the nasal bones and the LLC; if they become separated, the middle third of the nose can collapse causing nasal obstruction and an undesirable external appearance of the nose ${ }^{(2)}$. The middle and the lower thirds of the nose play an important role as the nasal 
valve area. With increasing age, the overlap between the ULC and LLC becomes reduced, and loss of elasticity of the cartilages can result in their collapse, again causing nasal obstruction. In approximately $17 \%$ of the population, there is no overlap between ULC and LLC, the lack of support between these structures makes those individuals more liable to nasal impingement and obstruction on inspiration (2).

The nose has crucial functions in providing humification, heating and filtration of the inspired air. The nasal valve can be subdivided into the external and internal nasal valves. The external nasal valve is formed by the nasal floor inferiorly, the nasal septum and medial crura medially, the alar cartilage and fibrofatty tissue anterolaterally, and the internal nasal valve opening posteriorly ${ }^{(3)}$. The internal nasal valve is the aperture between the nasal septum and the ULC. The angle formed between them is approximately $10-15^{\circ}$ in the Caucasian population ${ }^{(4)}$. Airway resistance during breathing is important for optimal lung expansion. The nose is responsible for $30-50 \%$ of the airway resistance during inspiration ${ }^{(5)}$, the internal and external nasal valves provide such a resistance and acts as a flow limiter, in particular, the internal nasal valve is the major flow limiting segment. When either of the two areas are narrowed, nasal obstruction occurs. As per Poiseuille's Law, resistance is inversely proportional to the radius to the fourth power, therefore even a small narrowing can have a significant impact on airflow resistance. Inspiratory air currents are directed upwards across the inferior and middle turbinates and thereafter into the nasopharynx. The direction of air flow is partly governed by the horizontal position of the anterior nares and the shape of the septum. The cartilaginous skeleton of the nasal alae prevent their collapse during negative pressure inspiration.

The shape of the nasal tip is determined by the strength and shape of the LLC and the thickness of the overlying skin ${ }^{(6)}$. It has been argued that the tip is the most important determinant of a beautiful nose ${ }^{(7)}$. A bulbous tip can be due to convex LLC and/ or thicker skin over the tip, it can be corrected by trimming of the subcutaneous fat and cephalic trim for convex LLC. However, care needs to be taken as excessive cephalic trim, can lead to depressed LLC, causing the external nasal valve to collapse, resulting in signs and symptoms of nasal obstruction ${ }^{(6)}$. Clearly, this highlights the delicate balance between functionality and cosmesis of the nose that needs to be taken into consideration when performing a rhinoplasty.

The ideal width of the nose measure as the alar base should be one fifth of the total width of the face (see later). A large tip lobule and wide alar bases can both give the appearance of a disproportionately large nose from an anterior view. A wider alar base with flared alae are particularly common in Southeast

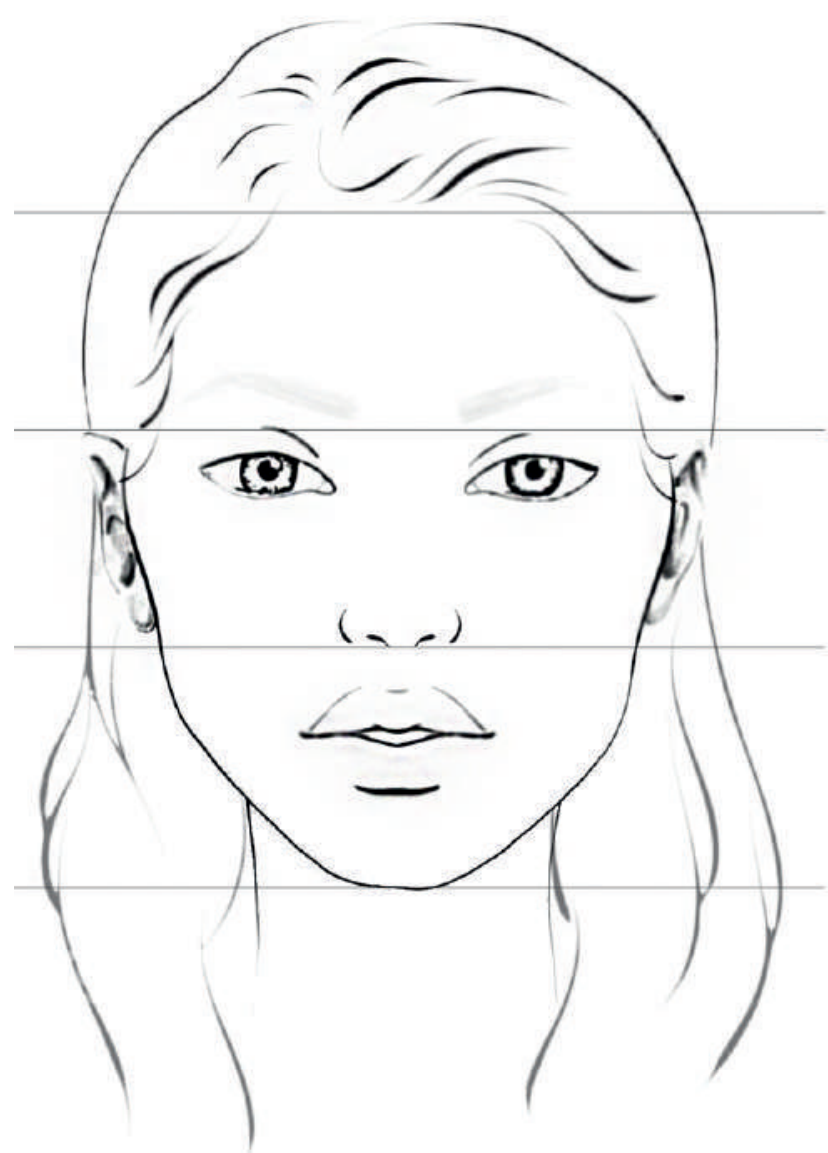

Figure 1. Horizontal thirds of the face according to neoclassical canon.

Asian ${ }^{(7)}$ and Afro-Caribbean noses ${ }^{(8)}$. This can be correct via a wedge incision to narrow the base. However, as the nasal aperture is a component of the external nasal valve, excessive narrowing of the aperture can again lead to iatrogenic nasal obstruction.

\section{Horizontal thirds}

In his publications on the human anatomy, Da Vinci divided the face into equal horizontal thirds (Figure 1). The upper third measures from the trichion (midpoint of the hairline) to the glabella (area above the nose and between the eyebrows). The middle third measures from the glabella to the subnasale (where nasal septum meets the upper lip). The lower third measures from the subnasale to the menton (most inferior point of the chin). As the nose occupies the middle third of the face, the ideal nose length should be in proportion to the midface. This has been proposed to be $0.67 \times$ midfacial height ${ }^{(9)}$. Milutinovic et al. examined photographs of Caucasian female celebrities (actresses and models) and compared them to a group of anonymous Caucasian females from the general population. They found anonymous Caucasian females showed statistically significant differences between the sizes of the three horizontal thirds, whereas the female celebrities (named as most beautiful faces by fashion 


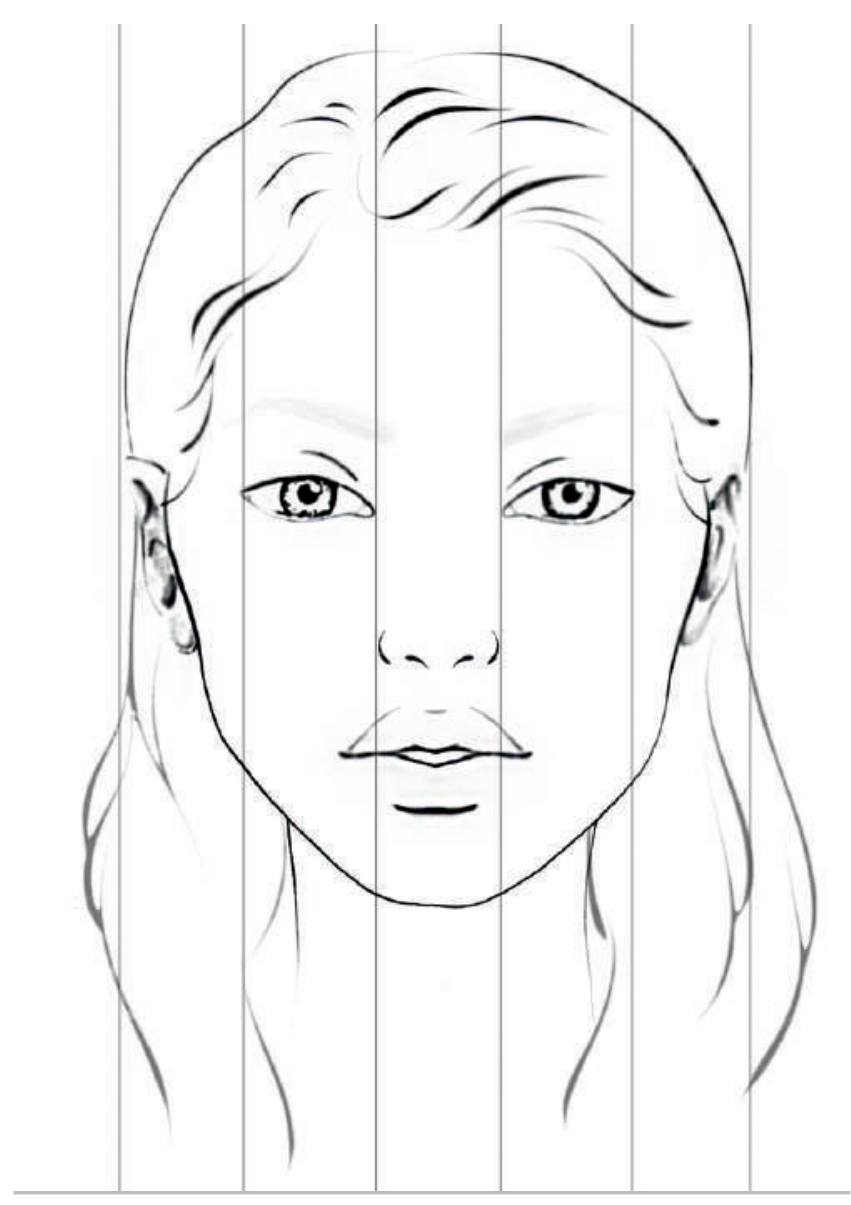

Figure 2. vertical fifths rule of the face according to neoclassical canon.

magazines) showed no significant difference between the sizes of their facial thirds ${ }^{(10)}$. Therefore, the relationship between neoclassical norm of equal thirds and the modern perception of beauty appears to hold.

It is worth noting that the method of thirds is based on Greek neoclassical canons and typically applies to Caucasian populations, but interestingly demonstrates consistent differences between ethnicities. Sim et al. looked at facial proportions of southern Chinese females and found the middle third to be consistently taller in the Chinese population compared to the Caucasian population, whilst the upper third (forehead) was smaller (11). It has been shown that the greatest interethnic variability in facial thirds exist in the height of the forehead ${ }^{(12)}$, and therefore, in apply the rule of thirds, one must take into account the ethnic background of the patient.

\section{Vertical fifths}

In the vertical plane, the neoclassical canon divides the face into equal fifths. The lateral fifth either side extends from the lateral helix of the ear to the exocanthus of the eye. The two eye fissures represent one fifth each. The middle fifth, which is the distance between the medial canthi of the eye, corresponds to the width of the nose (Figure 2), as measured between the alae bilaterally. Therefore the 'perfect' nose conforming to the vertical fifth rule should be one fifth of the width of the face. As is the case with horizontal thirds, there are deviations from this rule based on ethnicity, and this rule may be considered too narrow for many faces, especially in non-Caucasian populations. The stereotypical African-American nose has a wider nasal base (8). Similarly, Sim et al. noted that Chinese women had wider intercanthal distances and wider nasal base ${ }^{(11)}$. Interestingly, this preserves the vertical harmony of the face as the wider intercanthal distance complements the wider nasal base, making the face appear in proportion still.

\section{Golden ratio}

The concept of the golden ratio dates back to Ancient Greece. It was named as Phi by the sculptor Parthenon Phidias and is an irrational number approximately equal to 1.618 . It is also known as the Fibonacci ratio or the 'divine proportions'. The golden ratio is naturally occurring, and widely used in architecture and art but has also been applied to facial aesthetics. Proportions conforming to this ratio are said to be aesthetically pleasing to the eye and our brains subconsciously attach the concept of beauty to it. The value has been applied to many facial proportions. Of note, it describes the ideal mouth width to nose width ratio ${ }^{(13)}$.

\section{Dorsum}

The profile of the nose is a vital consideration in terms of its overall aesthetic appearance. There are variations of an attractive dorsum. A straight dorsum between the radix (depression at the root of the nose) and the tip with no supratip break, or a straight dorsum reduced to a level approximately $2 \mathrm{~mm}$ below the tip, creating a retroussé with a supratip break are both considered attractive ${ }^{(9)}$. The latter was preferred by Caucasian females. However, an over-section of the dorsum resulting in excessive concavity has become a hallmark of an operated nose, which is a look most patients want to avoid. Hence there has been a possible shift towards preference for a straight dorsum ${ }^{(14)}$. This interestingly, highlights the concept that aesthetic surgery can incite changes in beauty standards, something that may become more prevalent in the coming years.

\section{Radix projection}

Radix projection- measured as vertical distance between the corneal and the radix plane, can be expressed on the basis of ideal nose length. Byrd and Hobar ${ }^{(9)}$ proposed that this should be $0.28 x$ the ideal nose length. The aesthetic range for Caucasian population is normally $9-14 \mathrm{~mm}$.

\section{Tip projection}

The tip projection of the nose should be proportional to the nasal length. This has been a topic of much debate and research, 

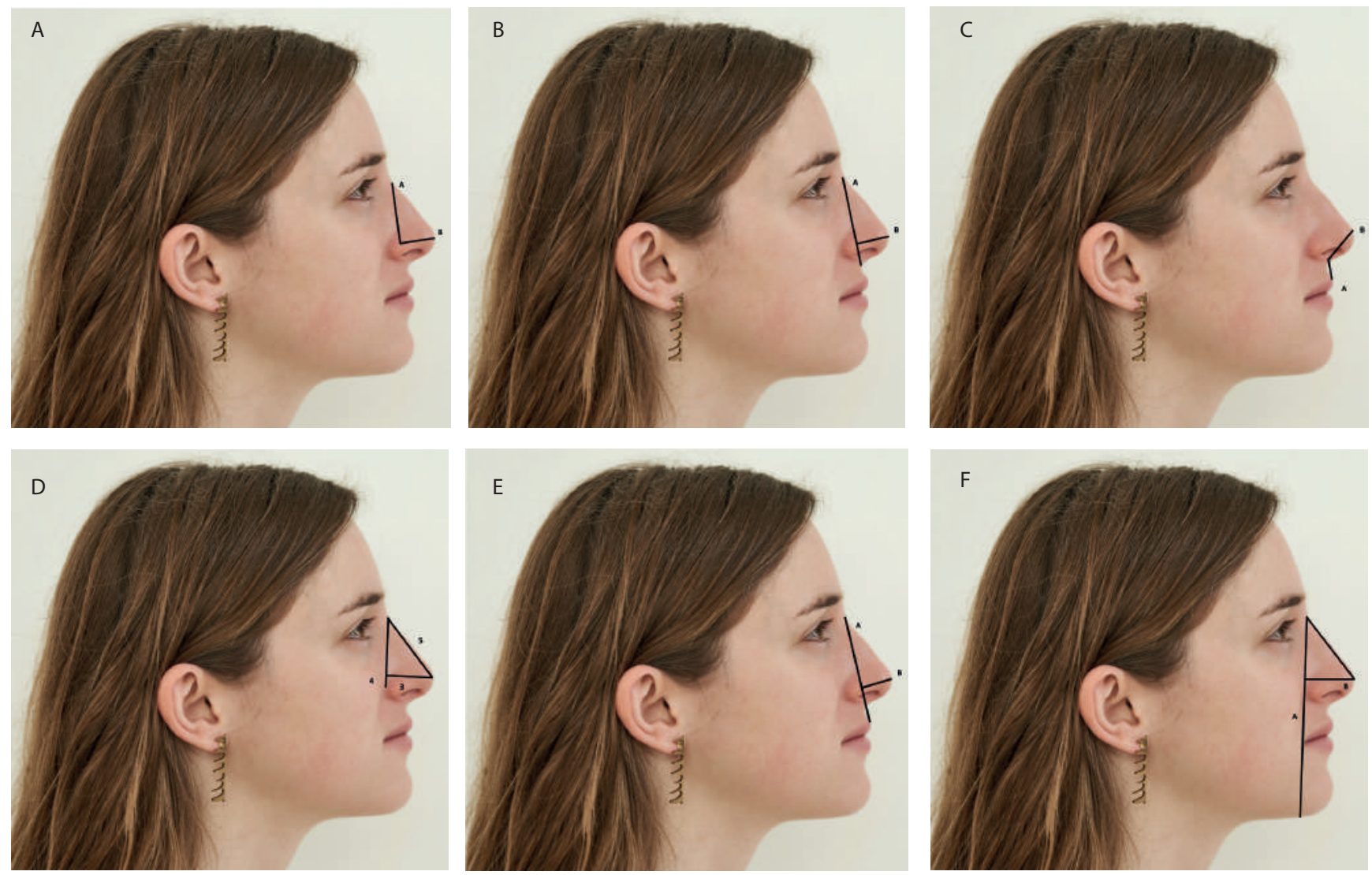

Figure 3. A) Baum's method: ratio of $A$ to $B$ should be $2: 1$. B) Powell and Humphrey's method: ratio of $A$ to $B$ should be $2.8: 1$. C) Simon's method: ratio of $A$ to $B$ should be $1: 1$. D) Goode's 3-4-5 triangle as illustrated. E) Crumley and Lanser's method: line B should be 0.2833 length of line $A$. F) Crumley and Lanser's other method as an extension of Goode's method: line B should be 0.2364 length of line A.

with various rules and calculations have been put forward over the years. Byrd and Hobar ${ }^{(9)}$ expressed the ideal tip projection as $0.67 x$ the ideal nasal length. Baum's method describes a 2:1 ratio of a vertical line from the nasofrontal angle to the vertex of the nasolabial angle that ends at a perpendicular junction to a horizontal line passing to the nasal tip (Figure 3a). This ratio was felt to produce excessive tip projection by Powell and Humphreys ${ }^{(15)}$, hence they modified this ratio to 2.8 : 1 by measuring the vertical line from the nasofrontal angle to the nasolabial angle (Figure 3b). A third method described by Simons ${ }^{(16)}$ proposed that the upper lip and the base length of the nose should be equal in a ratio of $1: 1$, hence incorporating consideration of the lip in determining the ideal nasal tip projection (Figure $3 c$ ). Goode's method uses a 3-4-5 triangle (Figure 3d). One side of the triangle is measured from the nasofrontal angle through to the alar crease; the second line is perpendicular to the first line going from the alar crease to the defining point of the nasal tip. The third line is drawn along the dorsum of the nose completing the right angle triangle. The ratio of the projection of the nose measured from the alar crease should be $0.55-0.60$ of the nasal dorsal length (measured from the nasion to the nasal tip) ${ }^{(15)}$. Lastly, in a study by Crumley and Lanser ${ }^{(17)}$, two more ratios were put forward. The first ratio uses the distance from the nasofrontal angle to the upper lip vermilion-cutaneous border, the ideal nasal projection should be 0.2833 anterior and perpendicular to this line (Figure 3e). The second method, put forward in the same paper, is similar to Goode's method but measures the vertical distance from the nasofrontal angle to the mandibular profile. The ideal tip projection should be 0.2364 of this vertical length (Figure 3f). The advantage of the Crumley and Lanser methods is that they take into account the upper lip length and the total facial height including the superior-inferior dimensions of the maxilla and the mandible. Interestingly, despite the various methods proposed, other research has suggested that none of the rules or proportions for nasal tip projection correlate with facial attractiveness, although the most attractive faces have nasal tip projections that are closer to the ideal ratios proposed by Goode and Crumley than unattractive faces ${ }^{(18)}$.

The potential issue with utilising the aforementioned ratios other than the Crumley and Lanser method is that they do not take into account the proportions of the lower third of the face, in particular, the mandible. The authors recommend using the Crumley and Lanser method in order to keep the nose in 


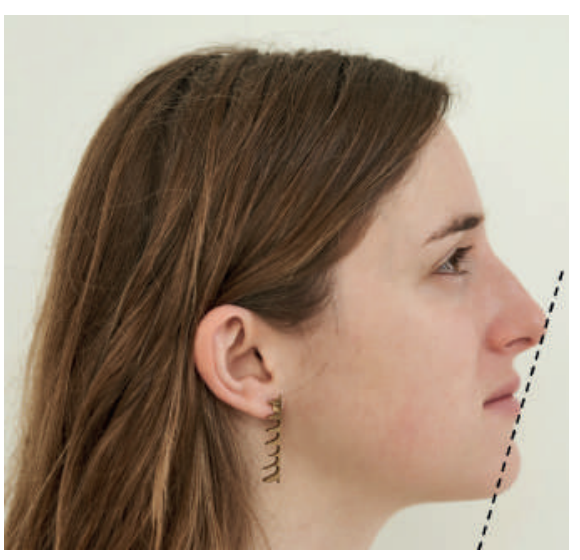

Figure 4. Nasomental line- the nasomental line connects the nasal tip to the lips and then the chin. The chin should deviate no more than $4 \mathrm{~mm}$ either side of the nasomental line to be considered attractive.

proportion to the overall balance of the face, which arguably gives superior cosmesis than a mathematically ideal nasal tip projection, but is not in harmony with the rest of the face. For example, a prognathic chin can make ones nose look bigger. This is a particular consideration in Asian patients, where the chin is typically shorter and more retruded ${ }^{(19)}$.

\section{Tip rotation}

Tip rotation is a contributing factor to an aesthetically pleasing nasal profile. A range of nasolabial angle from 95-115 degrees is considered desirable in women, whilst 90-95 degrees is preferred in men. In a study by Sinno et al., an angle of $104.9 \pm$ 4.0 degrees was preferable in women and $97 \pm 6.3$ degrees was preferable in men as rated by the general population ${ }^{(20)}$. The tip rotation should always be considered with the protrusion of the upper lip in mind; a greater lip protrusion may tolerate a greater degree of nasal tip rotation, such as that typically seen in African-Americans.

\section{Columellar show}

The columella is the bridge of tissue that separates the nostrils at the nasal base. Columellar show is an assessment of how much of the inner lining of the nostrils is visible on profile view. The ideal columellar show is generally accepted to be between $2 \mathrm{~mm}-4 \mathrm{~mm}{ }^{(21)}$. Excessive columellar show can be due to a variety of reasons, such as natural tendency towards hanging columella and alar retraction, previous rhinoplasty, trauma, or a combination of factors. Nine alar- columella relationships exist due the three configurations each for the columella (normal, hanging, retracted), and the alar rim (normal, hanging, retracted). The relationship between the alar and the columella results in 3 types of excessive columellar show: upper, lower and combined. Out of those, upper show is the most common. Upper excessive columellar show occurs when levator ala nasi over-contracts, elevating and retracting the alar rim, giving the nostrils a more dilated appearance. Lower excessive columellar show occurs due to excessive downward pull of depressor septi nasi, making the nasal septum appear more pronounced in profile view. Combined columellar show is due to over-contraction of both aforementioned muscles.

\section{Nasomental line}

The nasomental line is one connecting the nasal tip to the menton, touching the lips (Figure 4). The aesthetics of the nose and the chin should harmonise. Ideally, the chin should lie along this line for no more than $3 \mathrm{~mm}$ posterior. Studies have found that deviance (either retrusion or protrusions) from the nasomental line of up to $4 \mathrm{~mm}$ have no real effect on attractiveness. However, deviance beyond this adversely impacts the aesthetic profile of the face ${ }^{(22)}$. Given the implication of the nasomental line, assessment of the nose should also take into consideration the size of the chin. Any patient contemplating rhinoplasty may also need correction of the chin to balance the overall facial proportions when seen in profile.

\section{Recent changes and future research}

More recently, the role of the aforementioned neoclassical canons and geometrical rules have been challenged. A study of Italian models selected for a beauty contest showed that the middle third of the face was smaller than the upper and lower third; and the intercanthal width was smaller than the nose width ${ }^{(23)}$. Another recent study ${ }^{(24)}$, found that in Caucasian females, a mathematically averaged nose is more attractive. Rating of the composite nose created from 80 separate images was higher than that of the original images. Moreover, the closer an individual's nose shape resembles the average shape, the more attractive it is. Shifts in contemporary concepts of beauty drives the need to test these empirical formulae in depth and develop new canons, ideally using a three-dimensional approach, and taking account the overall balance of the face and also ethnic and cultural differences.

\section{Conclusions}

The 'perfect' nose blends harmoniously with other features of the face. A nose suitable for a given face depend on the gender and build, as well as the overall dimensions of the face, hence a mathematically ideal nose is not necessarily the 'perfect' nose for every face. Rhinoplasties are of huge popularity, regardless of ethnicity (25-27); as such there has been rigorous research into the ideal nasal geometry to help guide surgical planning. There is controversy about whether the neoclassical canons and geometrical rules are still the desired target but nevertheless they provide useful guidance in the assessment of the nose. There may not be one exemplary 'perfect' nose universally but rather we should allow for ethnic variations and individual preferen- 
ces. The changing contemporary views on facial plastic surgery should challenge us to further develop our understanding and develop potential new canons using more advanced threedimensional technologies.

\section{Acknowledgements}

The authors would like to thank CLM for consenting to have photographs taken, EM for the photography and CLM for kindly providing the illustrations.

\section{Authorship contribution}

$A D$ and $Y Z$ conceptualized the article. $A D$ and $Y Z$ wrote the manuscript. The final manuscript was read and approved by all authors.

\section{Conflict of interest}

The authors declare no conflict of interest.

\section{Ethics approval and consent to participate}

Not applicable

\section{Availability of data and materials}

Not applicable

\section{Funding}

There was no funding for this work.

\section{References}

1. Inc GVR. Rhinoplasty Market Size Worth $\$ 5.4$ Billion by 2026 | CAGR: 6.4\%: Grand View Research, Inc. [Internet]. [cited 2020 Jan 31]. Available from: https://www.prnewswire.com/news-releases/rhinoplasty-market-size-worth-5-4-billion-by-2026--cagr6-4-grand-view-research-inc-300973774 htm

2. Jones $\mathrm{N}$. The nose and paranasal sinuses physiology and anatomy. Adv Drug Deliv Rev. 2001 Sep 23;51(1):5-19.

3. Hamilton GS. The External Nasal Valve. Facial Plast Surg Clin N Am. 2017 May;25(2):17994

4. Ghosh A, Friedman O. Surgical Treatment of Nasal Obstruction in Rhinoplasty. Clin Plast Surg. 2016 Jan 1;43(1):29-40.

5. Geurkink N. Nasal anatomy, physiology, and function. J Allergy Clin Immunol. 1983 Aug $1 ; 72(2): 123-8$

6. Toriumi DM. Structure concept in nasa tip surgery. Oper Tech Plast Reconstr Surg. 2000 Nov;7(4):175-86.

7. Yap EC. Principles of Structural Rhinoplasty in South East Asian Noses. Philipp J Otolaryngol Head Neck Surg. 2014 Nov 30;29(2):41-4.

8. Patel AD, Kridel RWH. African-American rhinoplasty. Facial Plast Surg FPS. 2010 May;26(2):131-41.

9. Byrd HS, Hobar PC. Rhinoplasty: a practica guide for surgical planning. Plast Reconstr Surg. 1993 Apr;91(4):642-56.

10. Milutinovic J, Zelic K, Nedeljkovic N Evaluation of facial beauty using anthropometric proportions. ScientificWorldJournal. 2014;2014:428250. Published 2014 Feb 20. doi:10.1155/2014/428250

11. Sim RS, Smith JD, Chan AS. Comparison of the aesthetic facial proportions of southern
Chinese and white women. Arch Facial Plast Surg. 2000 Jun;2(2):113-20.

12. Fang F, Clapham PJ, Chung KC. A systematic review of interethnic variability in facial dimensions. Plast Reconstr Surg. 2011 Feb;127(2):874-81.

13. Moss JP, Linney AD, Lowey MN. The use of three-dimensional techniquesin facial esthetics. Semin Orthod. 1995 Jun 1:1(2):94-104

14. Bueller H. Ideal Facial Relationships and Goals. Facial Plast Surg. 2018 Oct;34(5):45865.

15. Powell N, Humphreys B. Proportions of the aesthetic face. Thieme-Stratton; 1984. 104 p.

16. Simons: Nasal tip projection, ptosis and supratip thickening. Ear Nose Throat J 1982;61:452-455

17. Crumley RL, Lanser M. Quantitative analysis of nasal tip projection. The Laryngoscope. 1988 Feb;98(2):202-8

18. Devcic Z, Rayikanti BA, Hevia JP, Popenko NA, Karimi K, Wong BJF. Nasal tip projection and facial attractiveness. The Laryngoscope. 2011;121(7):1388-94

19. Zhang C, Zhao L, Li C, Shim Y, Cao D Advancing and Lengthening Genioplasty in Contouring of the Receding and Short Chin. J Craniofac Surg. 2017 Mar;28(2):314-317.

20. The ideal nasolabial angle in rhinoplasty: a preference analysis of the general population. Plast Reconstr Surg. 2014;134(02):20110.

21. Turkmani MG, De Boulle K. Excessive columellar show: causes, presentations and a new therapeutic approach with onabotulinum toxin A. Dermatol Surg Off Publ Am Soc Dermatol Surg Al. 2013 Mar;39(3 Pt 1):464-7.

22. Naini FB, Donaldson ANA, McDonald F, Cobourne MT. Assessing the influence of chin prominence on perceived attractiveness in the orthognathic patient, clinician and layperson. Int J Oral Maxillofac Surg. 2012 Jul;41(7):839-46.

23. Torsello F, Mirigliani L, D'Alessio R, Deli R. Do the neoclassical canons still describe the beauty of faces? An anthropometric study on 50 Caucasian models. Prog Orthod. 2010;11(1):13-9.

24. van Zijl FVWJ, Perrett DI, Lohuis PJFM, Touw CE, Xiao D, Datema FR. The Value of Averageness in Aesthetic Rhinoplasty: Humans Like Average Noses [published online ahead of print, 2020 Jan 21]. Aesthet Surg J. 2020;sjaa010. doi:10.1093/asj/sjaa010

25. ASAPS-Stats2018.pdf [Internet]. [cited 2020 Feb 3]. Available from: https://www.surgery. org/sites/default/files/ASAPS-Stats2018.pdf

26. Ishii $\mathrm{CH}$. Current update in asian rhinoplasty. Plast Reconstr Surg Glob Open. 2014;2(4):e133. Published 2014 May 7. doi:10.1097/GOX.0000000000000081

27. Momoh AO, Hatef DA, Griffin A, Brissett AE. Rhinoplasty: The African American Patient. Semin Plast Surg. 2009 Aug;23(3):223-31.
A. Ding
Academic Foundation Doctor Imperial College Healthcare NHS Trust St Mary's Hospital Praed Street London W2 1NY
United Kingdom

ISSN: 2589-5613 / ( 2020 The Author(s). This work is licensed under a Creative Commons Attribution 4.0 International License. The images or other third party material in this article are included in the article's Creative Commons license, unless indicated otherwise in the credit line; if the material is not included under the Creative Commons license, users will need to obtain permission from the license holder to reproduce the material. To view a copy of this license, visit http://creativecommons.org/ licenses/by/4.0/ 